\title{
The management of ectopic pregnancies and pregnancies of unknown location
}

Published online: 2 April 2004

(C) Springer-Verlag Berlin / Heidelberg 2004

\begin{abstract}
Since the introduction of dedicated Early Pregnancy Units and the use of high-resolution transvaginal probes ectopic pregnancies are diagnosed at earlier gestations. As a result, the treatment options in the management of ectopic pregnancies have diversified. In this review, the role of transvaginal sonography in the management of women with ectopic pregnancies is described and the different treatment modalities available in their management are critically evaluated. We assert that ectopic pregnancy should be diagnosed on the basis of positively visualising an adnexal mass using transvaginal sonography, rather than the absence of an intrauterine pregnancy. If a pregnancy cannot be seen, either inside or outside the uterus, this should be described as a pregnancy of unknown location and managed expectantly until the outcome is confirmed.
\end{abstract}

Keywords Ectopic pregnancy · Pregnancy of unknown location · Transvaginal sonography · Discriminatory zone $\cdot$ hCG $\cdot$ Progesterone

\section{Introduction}

Over recent years the incidence of ectopic pregnancy has remained static, 11.1 per 1000 pregnancies, and almost 32,000 ectopic pregnancies are diagnosed annually in the UK [1]. The morbidity and associated mortality of ectopic pregnancy have substantially decreased over the past 25 years; however, there were 13 maternal deaths resulting from ectopic pregnancies in the UK during the period 1997-1999 [1]. Ectopic pregnancy was the fourth leading cause of direct maternal deaths in the UK in this period, accounting for $80 \%$ of first trimester deaths [1]. With the advent of Early Pregnancy Units (EPUs) and the use of high-resolution transvaginal probes, the number of

G. Condous $(\bowtie) \cdot$ E. Okaro $\cdot$ T. Bourne

Gynaecological Ultrasound and Minimal Access Surgery Unit,

St. George's Hospital Medical School,

Cranmere Terrace, Tooting London, SW17 0RE, UK

e-mail: gcondous@hotmail.com stable ectopic pregnancies diagnosed has significantly increased and consequently treatment modalities have become less radical. The evolution of treatment has progressed from salpingectomy at the time of laparotomy to salpingostomy with conservation of the fallopian tube performed by laparoscopy. More recently, medical management in the form of systemic methotrexate and even expectant management have been adopted in select cases.

Clinical history and examination are often not helpful in women who present with lower abdominal pain and vaginal bleeding. With the use of high-resolution transvaginal ultrasound and sensitive immunoassays for human chorionic gonadotrophin (hCG), early visualisation and diagnosis of an ectopic gestation is possible. The presence of an intrauterine sac does not always rule out an ectopic pregnancy. Spontaneous heterotopic pregnancies are rare $(1: 10,000-1: 50,000)$ but should be considered in women with assisted conceptions as the incidence is as high as $1 \%$ [2]. One should always thoroughly visualise the adnexa even when an intrauterine sac has been seen in such women.

The diagnosis of ectopic pregnancy should not be based on an inability to visualise an intrauterine pregnancy, but rather by the positive visualisation of an adnexal mass using transvaginal sonography (TVS). If a pregnancy cannot be seen using TVS, then it is classified as a pregnancy of unknown location (PUL), $10 \%$ of which are ectopic pregnancies. One should visualise between 87 and $93 \%$ of ectopic pregnancies using TVS prior to surgery $[3,4]$. Misdiagnosis should be a rare event with the use of TVS. An EPU standard of care can be judged by its false-positive and false-negative rates for the diagnosis of ectopic pregnancy; however, as fewer women with a positive diagnosis undergo laparoscopy, this becomes more difficult to determine.

The ultrasonographic appearances of ectopic pregnancies are highly variable. Classically, a hyperechoic ring around the gestation sac in the adnexal region is described as the "bagel sign". More often, ectopic pregnancies are seen as a small inhomogeneous mass adjacent to and moving separate from the ovary [5]. Brown and 
Doubilet [6] pooled data from ten published studies and concluded that the most appropriate TVS criterion on which to diagnose ectopic pregnancy is any non-cystic adnexal mass (inhomogeneous mass). The presence of this inhomogeneous mass leads to a positive predictive value of $96.3 \%$, negative predictive value of $94.8 \%$, specificity of $98.9 \%$ and sensitivity of $84.4 \%$. This performed better than the visualisation of an adnexal mass with an echogenic or "tubal" ring, or an adnexal cystic mass, or an embryo with or without a heartbeat in the adnexal region. Experienced operators will not be surprised by this as the vast majority of ectopic pregnancies that are seen using TVS appear as an inhomogeneous small mass or "blob" next to the ovary with no evidence of a sac or embryo.

The ultrasonographic appearances, the level of serum hCG and the patient's symptoms are all important factors in assessing suitability for medical management. The dimensions of the ectopic should be described, as should the presence of an embryo with or without a heartbeat. The amount of bleeding that has occurred should be commented upon by looking for blood in the pouch of Douglas, classically ground glass in appearance. The appearances of blood and clot as opposed to serous free fluid are quite different and should not be confused when using TVS.

The corpus luteum is a useful guide when looking for an ectopic pregnancy, as it will be on the ipsilateral side in over $85 \%$ of cases [7]. The so-called pseudosac should no longer be controversial. This sign is largely based on historical data and relates to the use of transabdominal ultrasonography. This is a misnomer and probably represents a fluid collection or debris in the cavity. With the use of high-resolution vaginal probes, it is possible to distinguish an early gestational sac from intra-cavity fluid, thus making misinterpretation less likely.

\section{$\overline{\text { Role of expectant management in ectopic pregnancy }}$}

Expectant management of ectopic pregnancy is an option in a select group of women. Ylostalo et al. managed 15\% of their ectopic pregnancies expectantly and observed spontaneous resolution in $64.6 \%$ [8]. Inclusion criteria were very strict and stipulated that the patient had to be stable, compliant, and with no evidence of haemoperitoneum on TVS or other signs of tubal rupture. Follow-up for these women involved ultrasound scans and measurements of serial serum hCG titres until they had fallen to $<15 \mathrm{IU} / 1$.

Resolution rates for expectant management of ectopic pregnancy were as high as $88 \%$ when initial serum hCG was less than $200 \mathrm{U} / \mathrm{l}$ - similar resolution rates were also seen when the initial serum hCG was less than $1000 \mathrm{U} / 1$ $[9,10]$.

In our unit, we offer expectant management to stable women with an initial serum hCG less than $1500 \mathrm{U} / \mathrm{l}$ and falling with an initial serum progesterone less than $20 \mathrm{nmol} / \mathrm{l}$. This accounts for only $10 \%$ of our ectopic population, with a resolution rate of $86 \%$. Expectant management requires very close follow-up and is reserved for select cases, with out of hours emergency back-up essential in the event of clinical deterioration.

\section{Medical management of tubal ectopic pregnancy}

Medical treatment with methotrexate is successful in 71$100 \%$ of cases [11]. It also performs well when compared with other standard treatment regimens. In a small randomised trial of unruptured tubal ectopic pregnancies (laparoscopic salpingotomy vs laparoscopic local methotrexate injection), successful resolution was seen in 80 90\% of cases [13]. Subsequent intrauterine pregnancy rates were 83.5 and $81 \%$, respectively [13]. Another randomised trial comparing systemic methotrexate or laparoscopic salpingostomy demonstrated comparable ipsilateral tubal patency rates (55 vs 59\%) [14].

Methotrexate can also be administered vaginally with ultrasonographic guidance, but it was less successful than laparoscopic salpingostomy in a randomised control trial [15]. When this method of methotrexate administration is compared with "blind" intra-tubal injection under laparoscopic guidance, its results are significantly better (RR 1.6, 95\% CI 1.0, 2.5) [16]. When comparing the administration route of methotrexate either transvaginally under sonographic guidance or systemically in a singledose intramuscular regimen, the combined results of three studies of small unruptured ectopic pregnancies showed no statistically significant difference in the primary treatment success (RR 1.2, 95\% CI 0.95, 1.5) $[17,18,19]$. Invasive techniques require a high degree of training and skill, and their use is not recommended given that single dose methotrexate is just as effective. In addition, systemic methotrexate provides significant cost savings when compared with laparoscopy [19, 20].

In the UK, $50 \mathrm{mg} / \mathrm{m}^{2}$ methotrexate is administered intramuscularly on day 1 . A second dose of methotrexate is required in up to $13 \%$ of cases if the hCG levels do not fall by $15 \%$ between days 4 and 7 [12]. In the U.S., $1 \mathrm{mg} /$ $\mathrm{kg}$ methotrexate is given on days 1,3 and 5 with folinic acid rescue on days 2,4 and 6. Resolution rates are comparable between the two regimens. It is important that there be no contraindications to the use of methotrexate; these include liver, renal or bone marrow impairment, or a viable pregnancy.

Successful outcome depends on the initial serum hCG levels (the likelihood of treatment failure is greater at higher serum hCG concentrations, i.e. $>5000 \mathrm{U} / \mathrm{l})$, the size of the ectopic pregnancy on TVS ( $<3 \mathrm{~cm}$ in diameter) and the presence or absence of fetal heart activity. In $10 \%$ of these women persistent trophoblastic disease is seen and tubal rupture can occur even with a falling serum hCG.

In our unit we offer medical management to women with tubal ectopic pregnancies who are clinically stable with a serum hCG of $<5000 \mathrm{U} / \mathrm{l}$, in whom the EP diameter is $<30 \mathrm{~mm}$ with no fetal heart activity and no signs of haemoperitoneum. The success rate is $88 \%$. 


\section{Surgical management of tubal ectopic pregnancy}

The laparoscopic approach has been conclusively shown to be better than laparotomy in the management of ectopic pregnancy [21, 22, 23]. The laparoscopic approach results in less haemorrhage and less pain. It is associated with a shorter hospital stay and recovery time. Laparoscopy is equally effective as open surgery in the treatment of tubal pregnancy [24], and considerably reduces costs [25]. Nevertheless, there will always be a place for laparotomy in those women who are haemodynamically unstable.

There is no consensus about which laparoscopic treatment modality (conservative linear salpingostomy vs radical surgery salpingectomy) preserves fertility best because no randomised control trials have been performed. Reported failure rates after conservative treatment vary from 3 to $29 \%[13,21]$, whereas there is practically no failure after radical treatment $(<0.5 \%)$. Salpingostomy is associated with higher subsequent intrauterine pregnancy (IUP; 61.4\%) and recurrent ectopic pregnancy rates (15.4\%) compared with salpingectomy (subsequent IUP rate $38.1 \%$ and the recurrent ectopic pregnancy rate $9.8 \%$ ) [24].

In women $<30$ years, subsequent fertility rates are the same for laparoscopic conservative and radical treatments [26]. Those women with a history of infertility do better with conservative treatment [26].

In women with no past history of tubal surgery or infertility and whose contralateral tube is normal, the fertility results after laparoscopic salpingectomy appear comparable to those observed after conservative laparoscopic treatment [27]. Conservative surgery should be performed when there is evidence of previous tubal infection at the time of laparoscopy. This confers the best chance for future fertility.

Ectopic pregnancy associated with haemoperitoneum on TVS suggests the possibility of tubal rupture and this situation requires surgical intervention. The incidence of haemoperitoneum ranges from 18 to $34 \%$ [28, 29].

In clinical practice the vast majority of ectopic pregnancies can be visualised using TVS [3, 4]. Consequently, the use of laparoscopy as a tool to diagnose ectopic pregnancy should be the exception rather than the rule.

In our unit, of those ectopic pregnancies treated surgically, $90 \%$ are performed by laparoscopy. If the contralateral tube is normal macroscopically, we perform a salpingectomy at the time of laparoscopy.

Anecdotally, if given the choice, women are very keen to conserve their fallopian tube at the time of surgery. To date, the psychological morbidity associated with conservative and radical surgery has not been prospectively evaluated.

\section{Non-tubal ectopic pregnancies}

Non-tubal ectopic pregnancies account for only 5\% of ectopic pregnancies, but they contribute a disproportionate number of serious complications. Their diagnosis may be difficult and they are associated with significant haemorrhage leading to a higher morbidity and mortality than tubal ectopic pregnancies.

Interstitial pregnancies account for about $2 \%$ of ectopic pregnancies [30]. The ultrasonographic appearance of an interstitial pregnancy is that of a bulge in the cornual region of the uterus where an extremely thin myometrial mantel surrounds the hyperechoic ring of the gestational sac [31]. The gestation sac is usually located more than $1 \mathrm{~cm}$ from the endometrial echo, although this is not mandatory. Hypoechogenic lesions situated in the cornual region may persist for 1 year, even following successful treatment and resumption of normal menstruation.

Cervical ectopic pregnancies are rare. The cervical appearance is classically barrel shaped and true cervical pregnancies are often relatively asymptomatic. It is important to differentiate this from an intact gestational sac passing through the cervix, which usually causes intense pain. Colour Doppler studies may assist in the diagnosis as they help to localise the uterine artery. This is a useful anatomical marker for the internal cervical os, below which the pregnancy implants. The presence of blood flow around the gestation sac is more suggestive of an implanted sac rather than one passing though the cervix. Ovarian pregnancy is also rare and has an incidence of 1:7000 deliveries and 1:34 ectopic pregnancies [32]. Diagnosis can be difficult, but the finding of an hyperechoic chorionic ring which moves with the ovary is highly suggestive.

Non-tubal ectopic pregnancies are a management problem. As the surgical approach is more hazardous, the mainstay of treatment in general is either systemic or local administration of methotrexate. In our unit we rely on a single-dose systemic methotrexate regimen for the treatment of non-tubal ectopic pregnancies and have rarely had problems with this approach. Injection of 50\% dextrose and methotrexate have been used to avoid major surgical intervention [33]. Similarly, systemic methotrexate and/or intra-tubal injection of potassium chloride has been used in unruptured interstitial pregnancies with complete resolution in 86.6-94\% [34, 35].

Abdominal pregnancies tend to be diagnosed later in pregnancy and are rare. The ultrasound features are well described and include the finding of an empty uterus separate from the fetus, placenta in an unusual location, no uterine mantle around the pregnancy or fetus and extreme oligohydramnios resulting in crowding of the fetal structures [36, 37].

Caesarean section scar pregnancy has been described only recently. The diagnosis is based on the visualisation of trophoblast located between the anterior uterine wall and the bladder [38]. Scar implantation should be further confirmed by applying gentle pressure on the cervix during a TVS. A gestational sac implanted outside the uterine cavity within the scar will remain in place during such a manoeuvre, whereas a cervical miscarriage will be easily displaced [39]. 


\section{Pregnancy of unknown location}

In $8-31 \%$ of women who present to an EPU, the pregnancy site is not visualised by TVS [40, 41]. These women are classified as having a "pregnancy of unknown location" (PUL). This is a descriptive term rather than a pathological entity. The varying prevalence may be attributable to the sonographer's ability. An inexperienced sonographer could potentially overlook some early intrauterine gestational sacs or adnexal masses, which in turn would result in a higher prevalence of PUL for a given EPU.

The PUL is diagnosed by TVS as there being no signs of either an intra- or extra-uterine pregnancy or retained products of conception in a woman with a positive pregnancy test (i.e. serum human chorionic gonadotrophin $(\mathrm{hCG})>5 \mathrm{U} / \mathrm{l})$. In this clinical situation there are three main outcomes: failing pregnancies (trophoblast in regression); early intrauterine pregnancies; or ectopic pregnancies that are too early to visualise on TVS. In a small number of cases there is no evidence of trophoblastic tissue on ultrasound or at laparoscopy and the serum hCG levels are low and have reached a plateau $(<500 \mathrm{U} / \mathrm{l})$. We have classified these as persisting PUL.

About 44-69\% [40, 41, 42] resolve spontaneously and are also known as trophoblast in regression [42]. It is important to note that the location of these pregnancies is never established and a proportion of these are resolving ectopic pregnancies that are never seen on TVS. Fourteen to $25.6 \%$ are subsequently diagnosed as ectopic pregnancies [41, 42].

Women with a PUL should be managed expectantly on the basis of measurements of serum levels of hCG and progesterone. This can be on an outpatient basis. Women thought to have a complete miscarriage at the initial scan should be managed as PULs. The diagnosis of complete miscarriage based on history and scan findings alone is unreliable, as up to $6 \%$ have an ectopic pregnancy [43]. If hCG levels do not fall, these women should be followed closely until the location of the pregnancy is confirmed.

Expectant management has been shown to be safe, reduce the need for unnecessary surgical intervention and is not associated with any serious adverse outcomes [40, $41,45]$. Nevertheless, $23-29 \%$ of these women require surgical intervention due to a worsening clinical condition or non-declining serum hCG $[40,41]$, and with experience, intervention rates can be as low as 9\% [44].

\section{Discriminatory zone and serial monitoring of serum hormonal levels}

When the location of a pregnancy cannot be confirmed on the basis of an ultrasound scan, the levels of serum hCG and progesterone and their interpretation should determine the management. An understanding of the pattern of serum hCG levels in early normal pregnancy and the correlation between low serum progesterone levels and the spontaneous resolution of a pregnancy are important hormonal variables in the management of pregnancies of unknown location; however, laparoscopy is still performed as a first-line investigation in some units. This is no longer acceptable practice.

The concept of combining ultrasound with measurements of serum hCG using a discriminatory zone is well described [46, 47, 48]. By correlating the serum hCG values to the size of an intrauterine gestational sac, a value can be chosen that corresponds to the threshold above which an intrauterine gestation sac should be seen. If a sac cannot be seen above the discriminatory zone, then steps must be taken to determine whether the pregnancy is abnormal or ectopic.

Barnhart et al. [46] showed that above a discriminatory level of $1500 \mathrm{IU} / 1$ an intrauterine gestation sac was seen in $91.5 \%$ of cases compared with $28.6 \%$ when levels were below $1500 \mathrm{IU} / \mathrm{l}$. In women without an ectopic mass or fluid in the pouch of Douglas, Mol et al. used a serum hCG cut-off of at least 2000 IU/1 [45].

However, it is noted that the discriminatory zone might vary among institutions due to the frequency of the probe used, different types of equipment and assay techniques. It is also dependent on operator experience. In early multiple pregnancies higher serum hCG titres are seen and this may lead to unnecessary concern about the location of the pregnancy.

A single measurement of hCG in practice will not be diagnostic in the majority of cases. When the serum hCG is above the discriminatory zone and an ectopic pregnancy is present, in most cases it will be large enough to be visualised by ultrasonography. Problems arise at lower serum hCG levels or in the smaller number of cases when an ultrasound diagnosis cannot be made. In such cases it is possible to distinguish between a PUL which will develop into a normal intrauterine pregnancy and those that subsequently become ectopic pregnancies on the basis of serum hCG increase over $48 \mathrm{~h}$. In normal intrauterine pregnancies there should be a $66 \%$ rise over the baseline value over $48 \mathrm{~h}$ [49]. Using this well-known algorithm is not without its pitfalls, as approximately $13 \%$ of ectopic pregnancies and $15 \%$ of normal intrauterine pregnancies screened in this way appear abnormal, giving contradictory results and delaying the diagnosis beyond $48 \mathrm{~h}$ [50]. It is possible to have either a "flourishing" ectopic pregnancy or a "sick" intrauterine-both can give conflicting results.

The vast majority of PULs are at low risk of ectopic pregnancy and in turn are made up of failing PUL/trophoblast in regression and IUPs. A failing PUL may be extra-uterine or intrauterine and generally will resolve spontaneously. A failing PUL is not necessarily a failing intrauterine pregnancy. These pregnancies are never seen on TVS, their baseline serum progesterone at presentation will be $<20 \mathrm{nmol} / \mathrm{l}$ and serial serum hCG levels fall. A baseline serum progesterone level of $<20 \mathrm{nmol} / \mathrm{l}$ will identify a failing PUL with a positive predictive value of $\geq 95 \%$ [44]. This compares favourably with complex multiparameter diagnostic models [44]. In contrast on- 
going IUP usually demonstrate a $>66 \%$ increase in serial serum hCG levels taken at 48 -h intervals.

Hormonal results should not be taken in isolation and the clinical assessment and subsequent ultrasound findings are essential to the ongoing management. When the diagnosis of ectopic pregnancy has been established (and this should be possible by TVS in the majority of cases), then appropriate medical or surgical management should be initiated. The overall rate of intervention for PUL managed expectantly in our series is $12.1 \%$, which is consistent with other groups [44].

\section{Persisting pregnancies of unknown location}

To date, there are no published data relating to persisting PUL. This small subset of women are defined as those where the serum hCG levels fail to decline, where there is no evidence of trophoblast disease, and the location of the pregnancy cannot be identified whether by ultrasound or laparoscopy. In general, the serum hCG levels are low $(<500 \mathrm{IU} / \mathrm{l})$ and have reached a plateau. We have treated nine such women successfully with methotrexate $50 \mathrm{mg} /$ $\mathrm{m}^{2}$ and their serum hCG levels subsequently resolved.

Care should be given before giving medical treatment for a PUL before the site of the pregnancy has been identified. A positive serum hCG does not always indicate pregnancy. Germ cell tumours may secrete hCG and should be considered, especially if a woman is adamant that she cannot be pregnant. In our unit, we have seen one malignant dysgerminoma of the ovary, one posterior cranial fossa germ cell tumour and one placental site trophoblastic tumour present in this way [51].

\section{Conclusion}

It is no longer acceptable to see an ultrasound report that states "an intrauterine sac cannot be seen, an ectopic pregnancy cannot be excluded". The diagnosis of ectopic pregnancy should be based on the positive visualisation of an extra-uterine pregnancy using TVS and not by the absence of an intra-uterine pregnancy.

The vast majority of ectopic pregnancies are still managed surgically. There is, however, great scope for the increased use of non-surgical management options, especially as increasingly more ectopic pregnancies are diagnosed in women who are stable and at lower levels of serum hCG. A select group of non-ruptured tubal ectopic pregnancies should be offered medical management in the form of methotrexate as it is safe and obviates the need for surgical intervention. Resolution is expected in up to $90 \%$ of these cases. A select few will be suitable for expectant management, with successful resolution seen in up to $88 \%$ of cases. Trials comparing expectant management to current practices are needed to evaluate its benefit or otherwise.

Expectant management of pregnancies of unknown location is safe when serum hCG and progesterone mea- surements are combined with serial TVS. This should not rule out performing a laparoscopy if there is a high index of suspicion of ectopic pregnancy based on clinical findings.

\section{References}

1. "Why Mothers Die" Triennial Report 1997-1999. Confidential Enquiry into Maternal Deaths, UK

2. Ludwig M, Kaisi M, Bauer O et al. (1999) Heterotopic pregnancy in a spontaneous cycle: do not forget about it! Eur J Obstet Gynecol Reprod Biol 87:91-93

3. Cacciatore B, Stenman UH, Ylostalo P (1990) Diagnosis of ectopic pregnancy by vaginal ultrasonography in combination with a discriminatory serum hCG level of 1000 IU/l (IRP). Br J Obstet Gynaecol 97:904-908

4. Shalev E, Yarom I, Bustan M, Weiner E, Ben-Shlomo I (1998) Transvaginal sonography as the ultimate diagnostic tool for the management of ectopic pregnancy: experience with 840 cases. Fertil Steril 69:62-65

5. Lerner J, Monteagudo (1995) Vaginal sonographic puncture procedures. In: Goldstein S, Timor-Tritsch IE (eds) Ultrasound in gynecology. Churchill Livingstone, New York, p 228

6. Brown DL, Doubilet PM (1994) Transvaginal sonography for diagnosing ectopic pregnancy: positivity criteria and performance characteristics. J Ultrasound Med 13:259-266

7. Jurkovic D, Bourne TH, Campbell S et al. (1992) The diagnosis of ectopic pregnancy using transvaginal color flow imaging. Fertil Steril 57:68-73

8. Ylostalo P, Cacciatore B, Koskimies A et al. (1991) Conservative treatment of ectopic pregnancy. Ann NY Acad Sci 626:516-523

9. Korhonen J, Stenman UH, Ylostalo P (1994) Serum human chorionic gonadotropin dynamics during spontaneous resolution of ectopic pregnancy. Fertil Steril 61:632-636

10. Trio D, Strobelt N, Picciolo C, Lapinski RH, Ghidini A (1995) Prognostic factors for successful expectant management of ectopic pregnancy. Fertil Steril 63:469-472

11. Parker J, Bisits A, Proietto AM (1998) A systematic review of single-dose intramuscular methotrexate for the treatment of ectopic pregnancy. Aust N Z J Obstet Gynaecol 38:145-150

12. Cobellis L (1998) Use of methotrexate in ectopic pregnancy. Results in 55 patients treated. Minerva Ginecol 50:513-517

13. Zilber U, Pansky M, Bukovsky I et al. (1996) Laparoscopic salpingostomy versus laparoscopic local methotrexate injection in the management of unruptured ectopic gestation. Am J Obstet Gynecol 175:600-602

14. Hajenius PJ, Engelsbel S, Mol BW, Van der Veen F, Ankum WM, Bossuyt PM, Hemrika DJ, Lammes FB (1997) Randomised trial of systemic methotrexate versus laparoscopic salpingostomy in tubal pregnancy. Lancet 350:774-779

15. Fernandez H, Yves Vincent SC, Pauthier S et al. (1998) Randomized trial of conservative laparoscopic treatment and methotrexate administration in ectopic pregnancy and subsequent fertility. Hum Reprod 13:3239-3243

16. Tzafettas J, Anapliotis S, Boucklis A et al. (1994) Transvaginal intra-amniotic injection of methotrexate in early ectopic pregnancy. Advantages over the laparoscopic approach. Early Hum Dev 39:101-107

17. Fernandez H, Bourget P, Ville Y et al. (1994) Treatment of unruptured tubal pregnancy with methotrexate: pharmacokinetic analysis of local versus intramuscular administration. Fertil Steril 62:943-947

18. Cohen DR, Falcone T, Khalife S et al. (1996) Methotrexate: local versus intramuscular. Fertil Steril 65:206-207

19. Yao M, Tulandi T, Kaplow M et al. (1996) A comparison of methotrexate versus laparoscopic surgery for the treatment of ectopic pregnancy: a cost analysis. Hum Reprod 11:2762-2766 
20. Mol BW, Hajenius PJ, Ankum WM, van der Veen F, Bossuyt PM (1997) Comparative costs of methotrexate and laparoscopic surgery. Hum Reprod 12:1603-1604

21. Murphy AA, Nager CW, Wujek JJ, Kettel LM, Torp VA, Chin HG (1992) Operative laparoscopy versus laparotomy for the management of ectopic pregnancy: a prospective trial. Fertil Steril 57:1180-1185

22. Vermesh M, Silva PD, Rosen GF, Stein AL, Fossum GT, Sauer MV (1989) Management of unruptured ectopic gestation by linear salpingostomy: a prospective, randomized clinical trial of laparoscopy versus laparotomy. Obstet Gynecol 73:400-404

23. Lundorff P, Thorburn J, Hahlin M, Kallfelt B, Lindblom B (1991) Laparoscopic surgery in ectopic pregnancy. A randomized trial versus laparotomy. Acta Obstet Gynecol Scand 70:343-348

24. Yao M, Tulandi T (1997) Current status of surgical and nonsurgical management of ectopic pregnancy. Fertil Steril 67:421-433

25. Mol BW, Hajenius PJ, Engelsbel S, Ankum WM, van der Veen F, Hemrika DJ, Bossuyt PM (1997) An economic evaluation of laparoscopy and open surgery in the treatment of tubal pregnancy. Acta Obstet Gynecol Scand 76:596-600

26. Job-Spira N, Bouyer J, Pouly JL, Germain E, Coste J, AubletCuvelier B, Fernandez H (1996) Fertility after ectopic pregnancy: first results of a population based cohort study in France. Hum Reprod 11:99-104

27. Dubuisson JB, Morice P, Chapron C, De Gayffier A, Mouelhi T (1996) Salpingectomy: the laparoscopic surgical choice for ectopic pregnancy. Hum Reprod 11:1199-1203

28. Saxon D, Falcone T, Mascha EJ, Marino T, Yao M, Tulandi T (1997) A study of ruptured tubal ectopic pregnancy. Obstet Gynecol 90:46-49

29. DiMarchi JM, Kosasa TS, Hale RW (1989) What is the significance of the human chorionic gonadotropin value in ectopic pregnancy? Obstet Gynecol 74:851-855

30. Kallchman GG, Meltzer RM (1966) Interstitial pregnancy following homolateral salpingectomy: report of 2 cases and review of the literature. Am J Obstet Gynecol 96:1139-1141

31. Jafrie SZ, Loginsky SJ, Bouffard JA et al. (1987) Sonographic detection of interstitial pregnancy. J Clin Ultrasound 15:253257

32. Rimes HG, Nosal RA, Gallagher JC (1983) Ovarian pregnancy: a series of 24 cases. Obstet Gynecol 61:174

33. Feichtinger W, Kemeter P (1987) Conservative treatment of ectopic pregnancy by transvaginal aspiration under sonographic control and methotrexate injection. Lancet 14:381-382

34. Benifla JL, Fernandez H, Sebban E et al. (1996) Alternative to surgery of treatment of unruptured interstitial pregnancy: 15 cases of medical treatment. Eur J Obstet Gynecol Reprod Biol 70:151-156

35. Jermy K, Condous G, Thomas J et al. (2003) Single-dose methotrexate in the treatment of cornual pregnancies diagnosed with ultrasound in the first trimester. Ultrasound Med Biol 29 (Suppl):S52

36. Hertz RH, Timor-Tritsch IE, Sokol RJ et al. (1977) Diagnostic studies and fetal assessment in advanced extrauterine pregnancy. Obstet Gynecol 50:63-65

37. Stanley JH, Horger EO III, Fagan CJ et al. (1986) Sonographic findings in abdominal pregnancy. Am J Radiol 147:1043-1046

38. Vial Y, Petignat P, Hohfeld P (2000) Pregnancy in a cesarean scar. Ultrasound Obstet Gynecol 16:592-593

39. Jurkovic D, Hillaby K, Woelfer B, Lawrence A, Salim R, Elson CJ (2003) First-trimester diagnosis and management of pregnancies implanted into the lower uterine segment Cesarean section scar. Ultrasound Obstet Gynecol 21:220-227

40. Hahlin M, Thorburn J, Bryman I (1995) The expectant management of early pregnancies of uncertain site. Hum Reprod 10:1223-1227

41. Banerjee S, Aslam N, Zosmer N et al. (1999) The expectant management of women with pregnancies of unknown location. Ultrasound Obstet Gynecol 14:231-236

42. Hajenius PJ, Mol BW, Ankum WM, van der Veen F, Bossuyt PM, Lammes FB (1995) Suspected ectopic pregnancy: expectant management in patients with negative sonographic findings and low serum hCG concentrations. Early pregnancy. 1:258262

43. Condous G, Okaro E, Khalid A, Alkatib M, Rao S, Bourne T (2003) Should complete miscarriages be followed up with serum human chorionic gonadotrophin levels? Ultrasound Obstet Gynecol 22 (Suppl 1):5

44. Banerjee S, Aslam N, Woelfler B et al. (2001) Expectant management of early pregnancies of unknown location: a prospective evaluation of methods to predict spontaneous resolution of pregnancy. Br J Obstet Gynaecol 108:158-163

45. Mol BW, Van der Veen F (2000) Results of expectant management of women early pregnancy and unknown location. Ultrasound Obstet Gynecol 15:265

46. Barnhart KT, Simhan H, Kamelle SA (1999) Diagnostic accuracy of ultrasound above and below the beta-hCG discriminatory zone. Obstet Gynecol 94:583-587

47. Kadar N, DeVore G, Romero R (1981) Discriminatory hCG zone: its use in the sonographic evaluation for ectopic pregnancy. Obstet Gynecol 58:156-161

48. Peisner DB, Timor-Tritsch IE (1990) The discriminatory zone of beta-hCG for vaginal probes. J Clin Ultrasound 18:280-285

49. Kadar N, Caldwell BV, Romero R (1981) A method of screening for ectopic pregnancy and its indications. Obstet Gynecol 58:162-166

50. Ling, Stovall (1994) Update on the diagnosis and management of ectopic pregnancy. Adv Obstet Gynecol 1:55-83

51. Condous G, Thomas J, Okaro E et al. (2003) Placental site trophoblastic tumour masquerading as an ovarian ectopic pregnancy. Ultrasound Obstet Gynecol 21:504-506 